\title{
The Legacy of E. Zavattini
}

\author{
Dimitar Bakalov
}

Institute for Nuclear Research and Nuclear Energy - Sofia - Bulgaria 


\section{Cargèse International School on QED, \& Quantum Vacuum, Low Energy Frontier - 2012}

2005: QED, Quantum Vacuum and the Search for New Forces, Les Houches 2000: Frontier tests of QED and physics of the vacuum, Trieste

1998: Frontier tests of QED and physics of the vacuum, Sandansky

1994:Workshop on physics of exotic atoms, Erice 


\section{The Legacy of E.Zavattini}

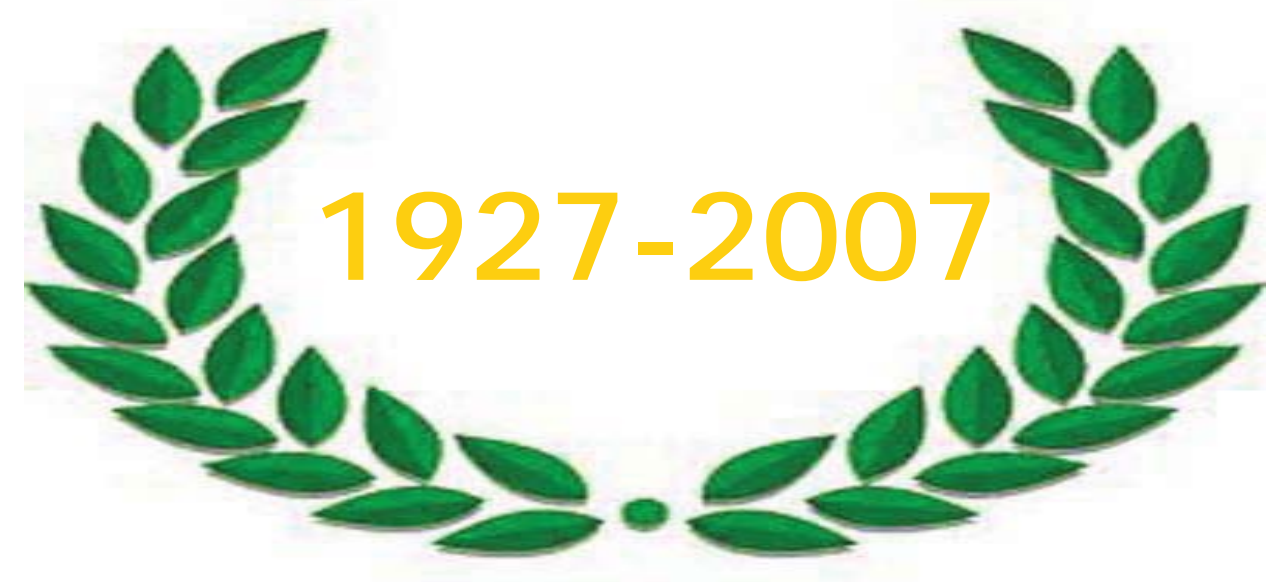




\section{The Legacy of E.Zavattini}

In the 1980's, in Dubna:

muon physics research, related to "muon catalyzed fusion".

E.Zavattini had already measured:

- muon capture rate

- muon life time

- cross sections with muonic atoms 


\section{The Legacy of E.Zavattini}

- Precision experiments, which:

- test fundamental theories

- are aimed at solving key problems

- display the interplay of weak, strong and EM interactions

- consider alternative models 


\section{Precision experiments}

Concluding remarks at the International

Conference on a European Hadron

Facility, Mainz 1986:

1. Muon capture rate and weak leptonhadron form factors $g_{A}$ and $g_{p}$

2. BNL proposal on the anomalous magnetic moment of the muon $a_{\mu}$. 


\section{Testing QED}

Particular interests:

Vacuum polarization and radiative effects:

- exotic atom spectroscopy

- muon anomaly measurements

- nonlinear QED effects 


\section{Of the Legacy of E.Zavattini:}

Three selected topics:

- Nuclear muon capture

- Nonlinear effects of QED

- Exotic atom spectroscopy 


\section{Muon capture by nuclei}

OMC: $\mu^{-}+p \rightarrow n+v_{\mu}$

$\mu^{-}+d \rightarrow n+n+v_{\mu}$

6 form factors at $q^{2} \sim 0.88 m_{\mu}{ }^{2}$

$g_{v}\left(q^{2}\right), g_{M}\left(q^{2}\right), g_{s}\left(q^{2}\right), g_{A}\left(q^{2}\right)$, $g_{T}\left(q^{2}\right), g_{p}\left(q^{2}\right)$

D. Bakalov, Cargèse 2012 


\section{Muon capture in $\mathrm{H}$ gas}

\section{First measurement of $\mu^{-}+p \rightarrow n+v_{\mu}$}

\section{Muon Capture in Gaseous Hydrogen}

The experimental result is $\Lambda_{\text {expt }}^{-}=651 \pm 57 \mathrm{sec}^{-1}$, which has to be compared with the theoretical rate $\Lambda_{s \text {, theor }}=626 \pm 26 \mathrm{sec}^{-1}$. From the experimental capture rate, and within the framework of the currently accepted theory, we have obtained for the induced pseudoscalar coupling constant $\left.g_{p}=-7.3 \pm 3.7\right) g_{V}$ The results of the present experiment are analyzed, together with results obtained from stopping negative muons in liquid hydrogen.

\section{To compare with: $\Lambda_{S}=725.0 \pm 17.4, g_{p}=7.3 \pm 1.1$ (MuCAP experiment, 2007)}




\section{Muon capture in liquid $\mathrm{H}$}

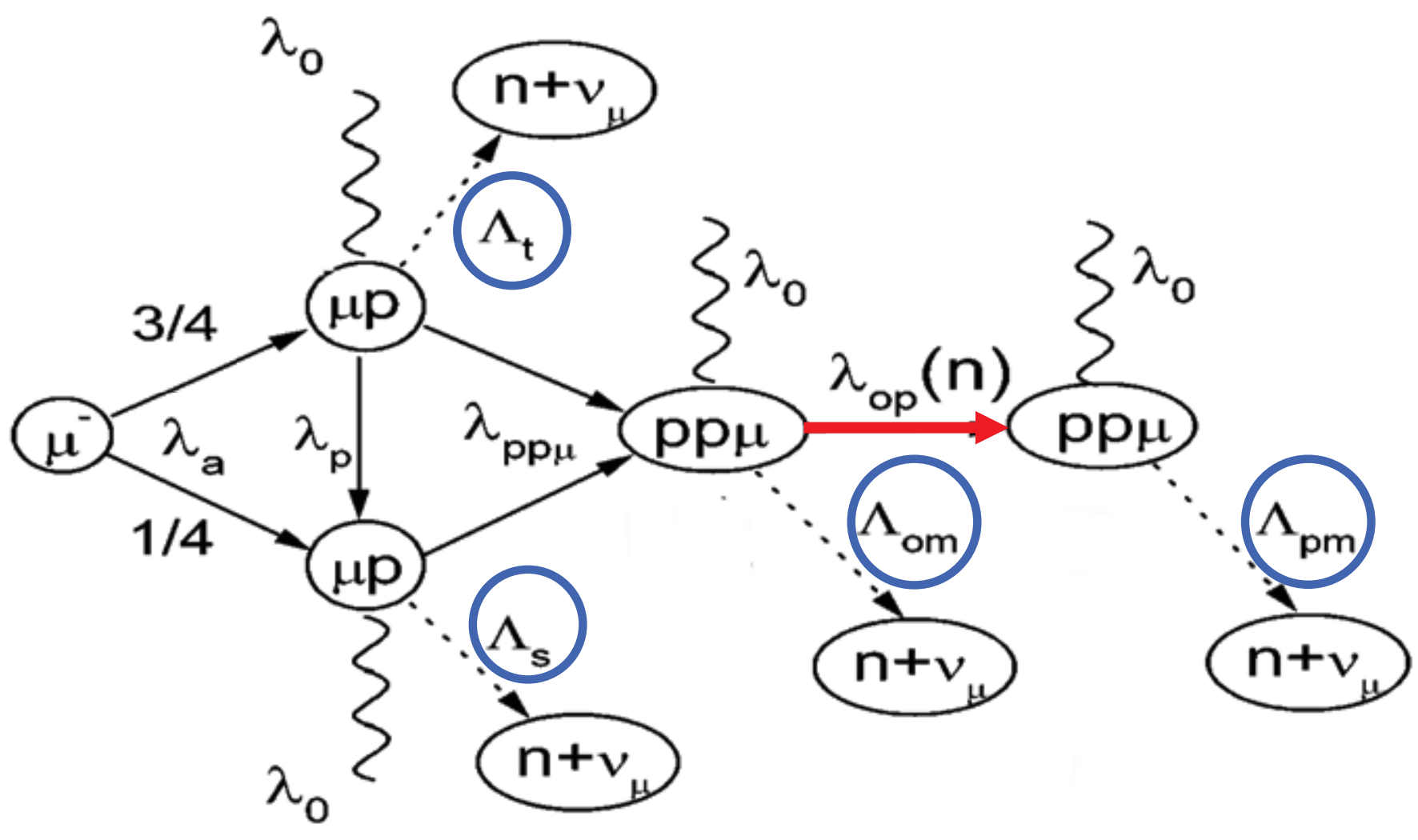

D. Bakalov, Cargèse 2012 


\section{Muon capture in liquid $\mathrm{H}$}

Nuclear Physics A352 (1981) 365-378 @ North-Holland Publishing Co., Amsterdam

\section{A NOVEL MEASUREMENT OF THE MUON CAPTURE RATE IN LIQUID HYDROGEN BY THE LIFETIME TECHNIQUE}

Abstract: The muon nuclear capture rate by protons has been measured by comparing the lifetime $\tau_{\mu}$ - of negative muons stopped in liauid hydrogen with the lifetime $\tau_{\mu}{ }^{+}$of positive muons. We get $\tau_{\mu^{-}}=2194.903 \pm 0.066 \mathrm{~ns}$ and $\tau_{\mu}{ }^{+}=2197.182 \pm 0.121 \mathrm{~ns}$. Taking into account also the previous determinations of $\tau_{\mu}{ }^{+}$, we present the updated determination of the muon lifetıme

I mproved to $\tau_{\mu+}=2197.078(0.073)$ ns [PLB137(1984)]

To compare with MuLAN (2007), similar method used:

$$
\tau_{\mu+}(\text { MuLan })=2196980.3(2.2) \text { ps. }
$$




\section{Ortho-para transitions in liquid $\mathrm{H}$}

Volume 104B, number 4

PHYSICS LETTERS

3 September 1981

MEASUREMENT OF THE ORTHO-PARA TRANSITION RATE IN THE $p \mu p$ MOLECULE AND DEDUCTION OF THE PSEUDOSCALAR COUPLING CONSTANT $g_{\mathrm{p}}^{\mu}$

The ortho-para transition rate in the p $\mu$ p molecule has been found experimentally to be $\lambda_{O P}=(4.1 \pm 1.4) \times 10^{4} \mathrm{~s}^{-1}$. Our recent result for the muon capture rate in liquid hydrogen can now be interpreted to extract the ortho-molecular capture rate: $\lambda_{\mathrm{OM}}=(531 \pm 33) \mathrm{s}^{-1}$. A deduction of the pseudoscalar coupling constant $g_{\mathrm{p}}^{\mu}$ is presented: we find $g_{\mathrm{p}}^{\mu}=8.7$ \pm 1.9 .

Theoretical calculations: $\lambda_{o p}=7.1 \pm 4.1 \times 10^{4} \mathrm{~s}^{-1}$ Still an open problem. 


\section{Nonlinear QED effects}

Vacuum birefringence in strong $B$ :

$\mathrm{n}_{\perp} \neq \mathrm{n}_{11}$

Leading to elipticity and rotation of linearly polarized light

(Analogous to Cotton-Mouton effect) 


\title{
Vacuum birefringence in $B$
}

Euler-Heisenberg theory:

$$
\begin{aligned}
L & =\left(E^{2}-B^{2}\right) /(8 \pi) \\
& +(A / 4 \pi)\left(\left(E^{2}-B^{2}\right)^{2}+7(E \cdot B)^{2}\right), A=O\left(\alpha^{2}\right)
\end{aligned}
$$

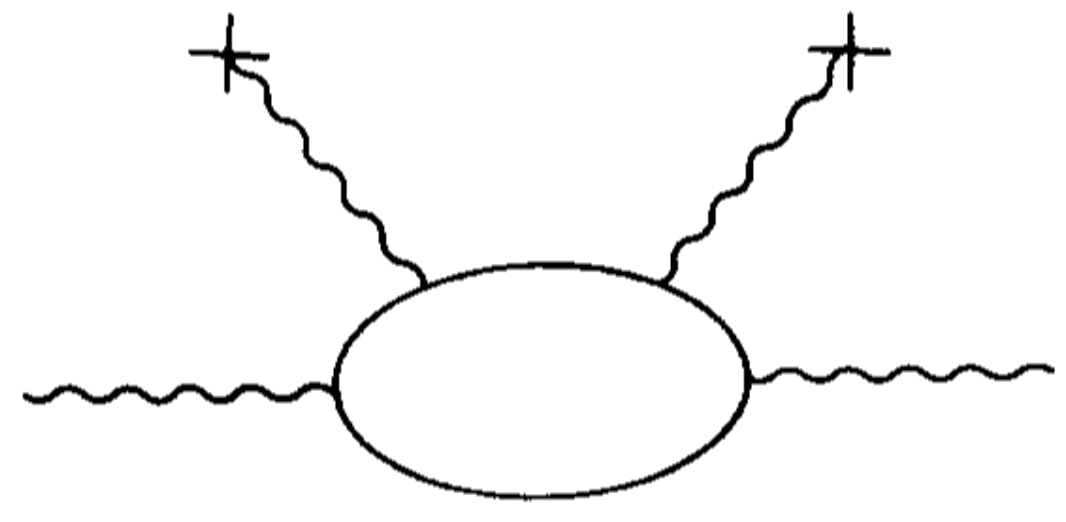

th.o.t.

\author{
D. Bakalov, Cargèse 2012
}




\section{Experimental method}

Volume $85 \mathrm{~B}$, number 1

PHYSICS LETTERS

30 July 1979

\section{EXPERIMENTAL METHOD TO DETECT THE VACUUM BIREFRINGENCE INDUCED BY A MAGNETIC FIELD}

\section{E. IACOPINI and E. ZAVATTINI}

CERN, Geneva, Switzerland

Received 28 May 1979

In this letter a method of measuring the birefringence induced in vacuum by a magnetic field is described: this effect is evaluated using the non-linear Euler-Heisenberg-Weisskopf lagrangian. The optical apparatus discussed here may detect an induced ellipticity on a laser beam down to $10^{-11}$.

D. Bakalov, Cargèse 2012 


\section{Vacuum birefringence in $B$}

\section{Other possible}

sources: axions

b)
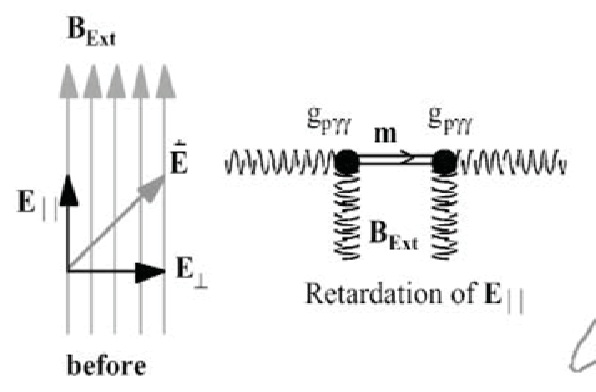

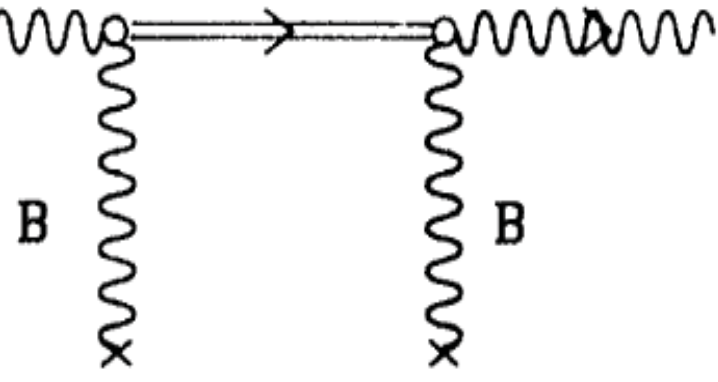

$\gamma$ mann a)
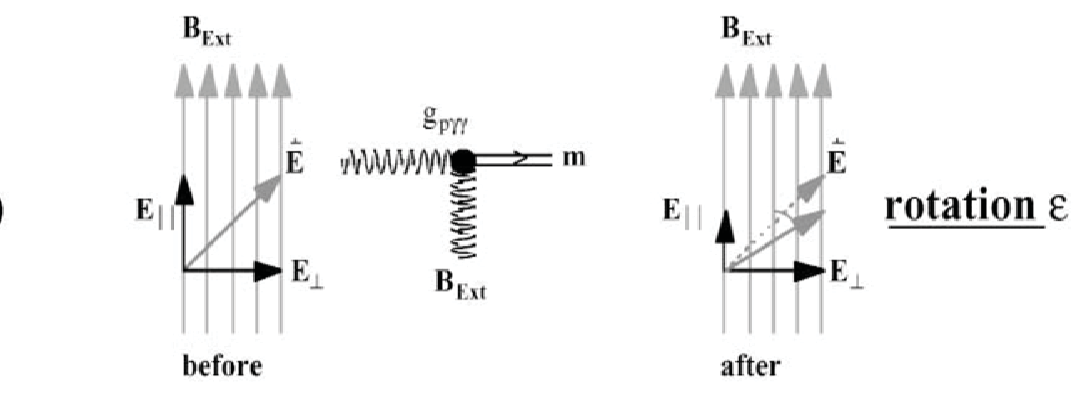
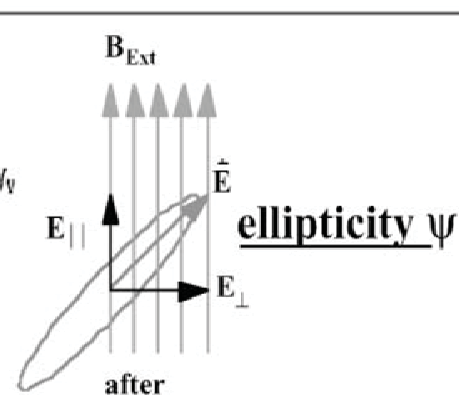

From: Phys.Rev.D47(1993)3707 [left], Nucl.Instrum.Meth. A461(2001)329 [right] 


\section{Search for "axions" (BFRT)}

PHYSICAL REVIEW D

VOLUME 47, NUMBER 9

\section{ARTICLES}

Search for nearly massless, weakly coupled particles by optical techniques
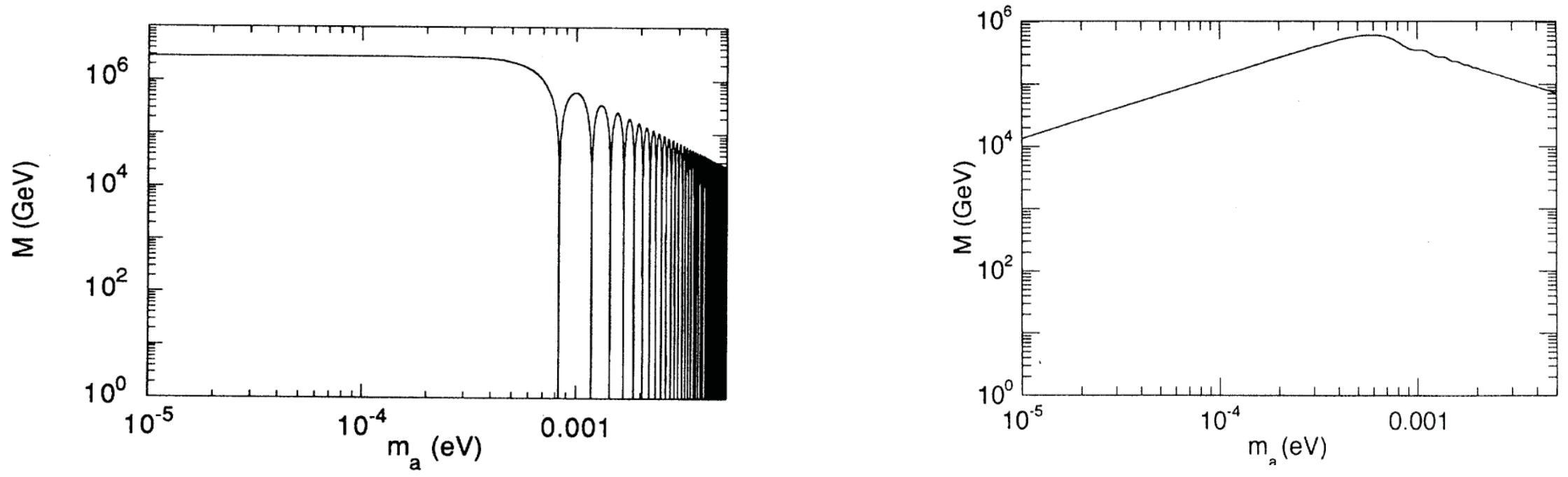

D. Bakalov, Cargèse 2012 


\section{Search for "axions" (PVLAS)}

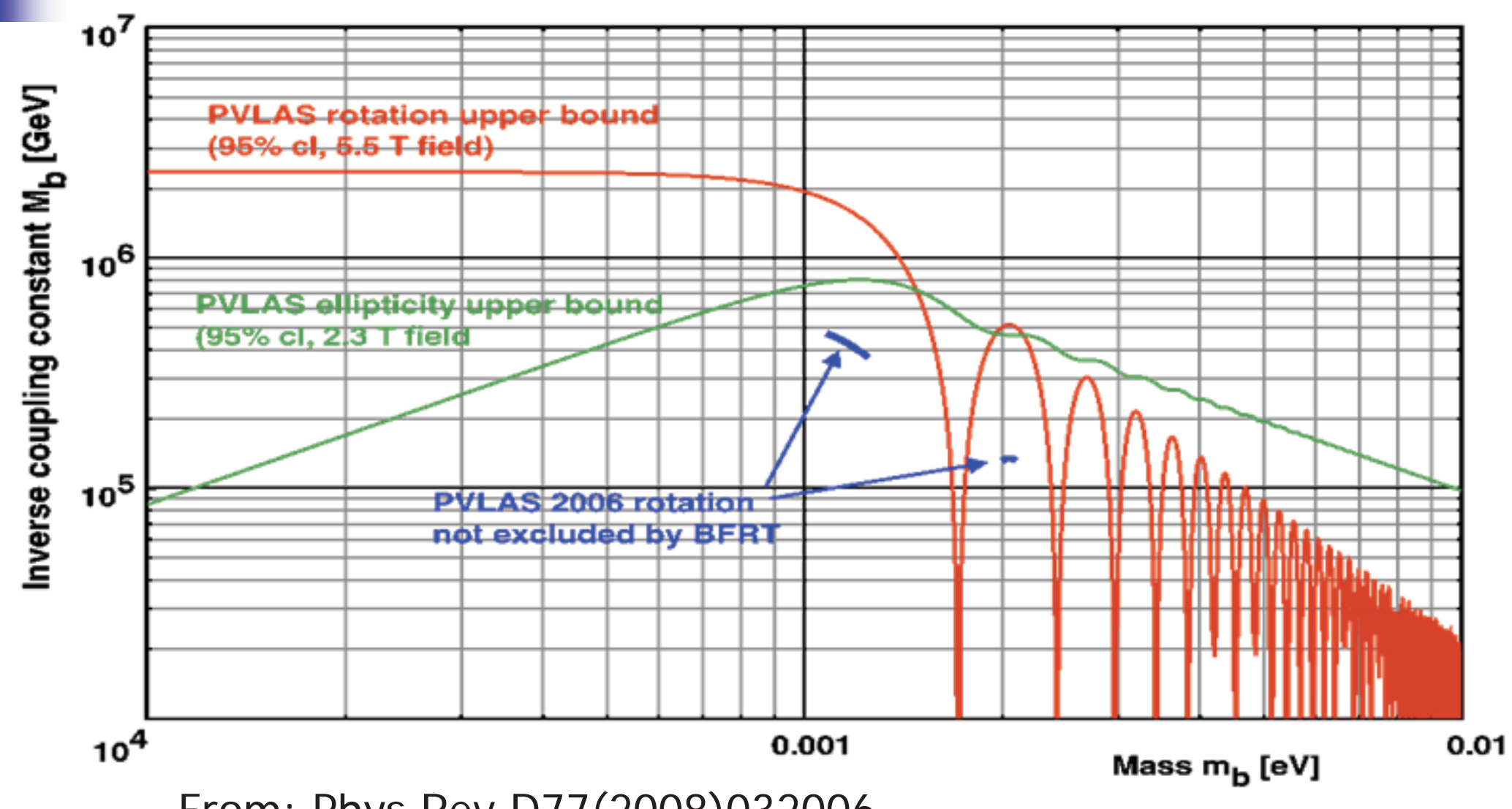

From: Phys.Rev.D77(2008)032006

D. Bakalov, Cargèse 2012 
Vacuum birefringence in $B$

-Vacuum birefringence in strong field: not yet observed

-Improved sensitivity experiments: in progress $(B M V, . .$.

-Search for weakly interacting nearly massless particles: in progress

-Photon regeneration experiments... 


\section{Exotic atom spectroscopy}

The electron replaced by a heavy charged particle.

$r \sim a_{0} /\left(m / m_{e}\right)$ : enhanced nucl.str.eff.

$E \sim E_{0} *\left(m / m_{e}\right)$ : different freq. range

- Kaonic atoms (DEAR)

- Antiprotonic atoms (Obelix)

- Muonic atoms (CERN, SIN) 


\section{Vacuum polarization in $(\mu \mathrm{He})^{+}$}

\section{$2 \mathrm{~S}_{1 / 2}-2 \mathrm{P}_{3 / 2}$ level difference}

Nuclear Physics A278 (1977) 381 - 386;

PRECISE MEASUREMENT OF THE $2 S_{\frac{1}{2}}-2 P_{z}$ SPLITTING IN THE $\left(\mu^{-4}\right.$ He) ${ }^{+}$MUONIC ION

Abstract: The results of a new measurement of the $2 S_{1 / 2}-2 \mathrm{P}_{3 / 2}$ splitting $S^{1}$ in the muonic ion $\left(\mu^{-4} \mathrm{He}\right)^{+}$ are presented. We found $S_{\mathrm{agp}}^{1}=1527.5 \pm 0.3 \mathrm{meV}$. Using the new, recently determined, value of the rms charge radius for ${ }^{4} \mathrm{He}$ we obtain for the difference $D$, between $S_{\text {erp }}^{1}$ and the corresponding theoretical prediction, the value $D=0.2 \pm 4.2 \mathrm{meV}$ : this value directly confirms, assuming $\mu-e$ universality, the QED vacuum polarization prediction to $0.25 \%$.

NOT reproduced by P. Hauser et al., PRA(1992) (low P) 


\section{Vacuum polarization in $(\mu \mathrm{He})^{+}$}

\section{$2 \mathrm{~S}_{1 / 2}-2 \mathrm{P}_{1 / 2}$ level difference}

Volume 73B, number 2

\section{PHYSICS LETTERS}

\section{MEASUREMENT OF THE $2 S_{1 / 2}-2 P_{1 / 2}$ SPLITTING IN THE $\left(\mu^{-4} \mathrm{He}\right)^{+}$MUONIC ION}

The measurement of the $2 S_{1 / 2} \rightarrow 2 P_{1 / 2}$ energy transition in muonic helium is presented. The energy difference $S^{1}$ is found to b $S_{\mathrm{gxp}}^{1}=1381.3 \pm 0.5 \mathrm{meV}$. This result agrees with the expected value $S^{1}=1381.2 \pm 0.3 \mathrm{meV}$ obtained assuming the previously measured value for the $2 S_{1 / 2} \rightarrow 2 P_{3 / 2}$ energy difference.

Confirmed by theory: 1381.716 meV (Martynenko 2006)

D. Bakalov, Cargèse 2012 


\section{Metastability of $(\mu \mathrm{He})^{+}{ }_{2 \mathrm{~S}}$ at $40 \mathrm{~A}$ ?}

Volume 33

29 JULY 1974

NUMBER 5

Measurement of the Initial Population and Decay Rate of the $\left(\mu^{4} \mathrm{He}\right)_{2 S}+$ System in a Helium Target at $50 \mathrm{~atm}$

Experimentally observed:

2S-fraction: $\sim 4 \%$

2S-lifetime: $\sim 1 \mu \mathrm{s}$ at $50 \mathrm{At}$.

D. Bakalov, Cargèse 2012 


\section{Metastability of $(\mu \mathrm{He})^{+}{ }_{2 \mathrm{~S}}$ at $40 \mathrm{~A}$ ?}

PHYSICAL REVIEW A

VOLUME 41, NUMBER 5

Formation and disappearance rates of metastable muon- $\alpha$ levels in high-pressure helium targets

Semi-quantitative theoretical model: prompt formation of excited clusters

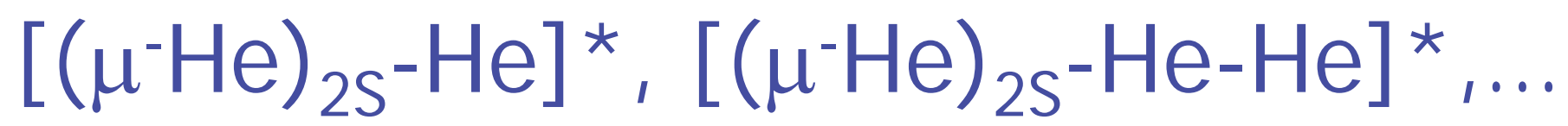




\section{Metastability of $(\mu \mathrm{He})^{+}{ }_{2 \mathrm{~S}}$ at $40 \mathrm{~A}$ ?}

But:

M.Eckhause et al., PRA33(1986) found:

at $40 \mathrm{At}$, the lifetime of $(\mu \mathrm{He})^{+}{ }_{2 S}$ is $40 \mathrm{~ns}$.

2S-metastability: an open problem.

Future experiments expected (PSI?) 


\section{Born-Infeld theory (1983)}

IL NUOVO CIMENTO

VoL. $78 \mathrm{~B}, \mathrm{~N} .1$

11 Novembre 1983

Vacuum Polarization Effects in the $\left(\mu^{-4} \mathrm{He}\right)^{+}$Atom and the Born-Infeld Electromagnetic Theory.

$L=b^{2}\left(1-\sqrt{ } 1-\left(E^{2}-B^{2}\right) / b^{2}-(E . B)^{2} / b^{4}\right)$

Lamb shift in $(\mu \mathrm{He})^{+}$can be reproduced by adjusting $b$

No vacuum birefingence produced 


\title{
Antiprotonic helium spectroscopy (Obelix Collaboration)
}

- Delayed annihilation of antiprotons stopped in Helium

- Dependence on contaminants

- Alternative mechanism suggested:

\author{
formation of $\left[(p-H e)^{+} \mathrm{He}\right]^{*}$ \\ ( not confirmed)
}




\section{Muonic hydrogen spectroscopy}

- Elastic scattering cross sections

- Muon transfer to heavier gases (xenon)

- Laser spectroscopy in excited states

- neavier elements muonic atoms

Hyperfine splitting in $(\mu \mathrm{H})_{1 s}$ ? 


\section{HFS in hydrogen-like atoms}

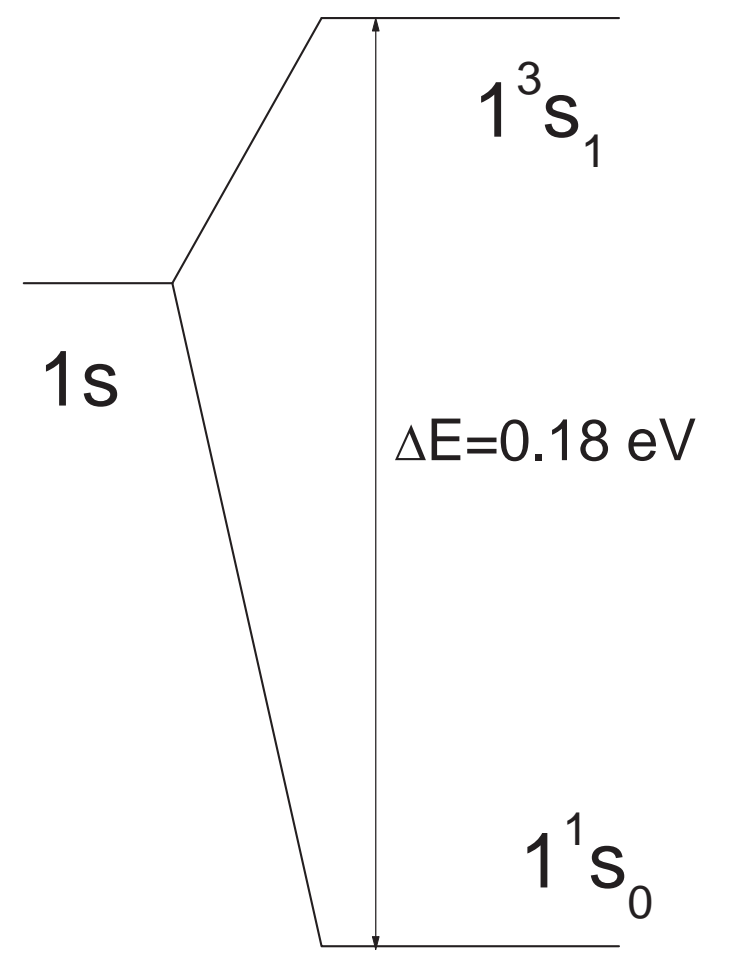

$$
\begin{aligned}
& \Delta \mathrm{E}=\Delta \mathrm{E}^{\mathrm{F}}\left(1+\delta^{\mathrm{QED}}+\delta^{\text {str }}\right) \\
& \delta^{\text {str }}=\delta^{\text {rigid }}+\delta^{\mathrm{pol}}+\delta^{\text {hvp }}+\ldots \\
& \delta^{\text {rigid }}=\delta^{\text {Zemach }}+\delta^{\text {recoil }} \\
& \delta^{\text {Zemach }}=-2 \alpha m \mathrm{R}_{\mathrm{p}}+\mathrm{O}\left(\alpha^{2}\right) \\
& \mathrm{R}_{\mathrm{p}}=<r>_{\mathrm{E} \text { om }} ; \mathrm{R}_{\mathrm{p}}{ }^{2} \neq \mathrm{r}_{\mathrm{sq}}{ }^{2} \\
& \Delta^{\text {pol }} \leftrightarrow \text { polarizability ' } \mathrm{a} \text { ' } ? \\
& \text { Two parameters }\left(\mathrm{a}, \mathrm{R}_{\mathrm{p}}\right) \text { from } \\
& \text { measurements in } \mathrm{H} \& \mu \mathrm{H} ?
\end{aligned}
$$

D. Bakalov, Cargèse 2012 


\section{Hyperfine splitting in $(\mu \mathrm{H})_{1 S}$}

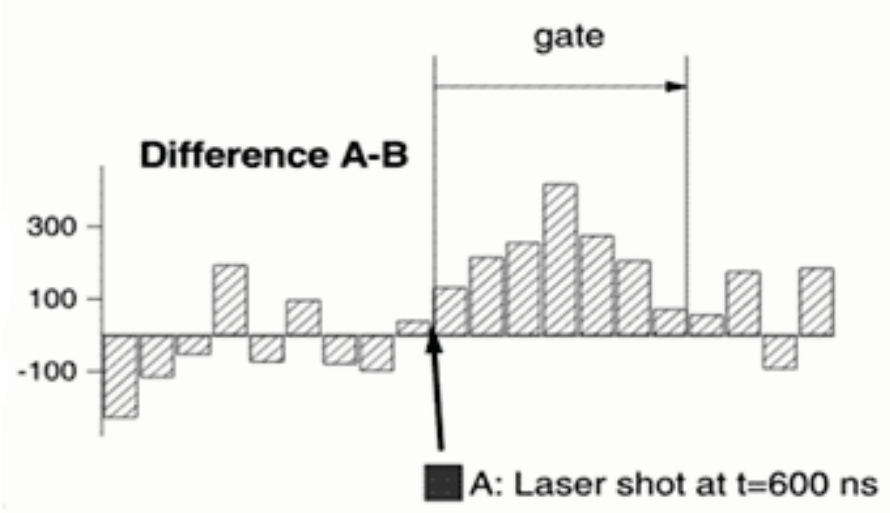

$\square$ B: No laser (background)

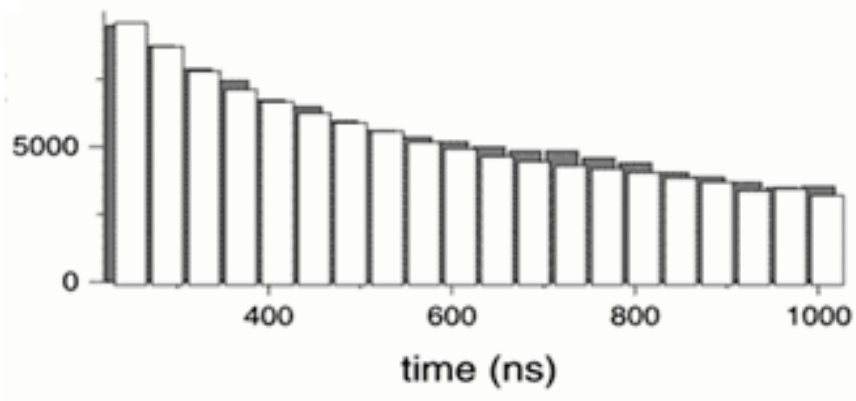

From: Hyperf.interact.136(2001)1
- Time distribution of the events of $\mu^{-}$transfer to: - walls of target volume - nuclei of appropriate heavier gas admixture - Tunable IR laser source

Nuclear Instruments and Methods in Physics Research A 341 (1994) Compact waveguide FEL for spectroscopic measurements in muonic hydrogen

\section{Bakalov, Cargèse 2012}




\section{The Legacy of E.Zavattini}

Ongoing experimental activities

- MULAN \& MUCAP

- Vacuum birefirngence

- Muonic helium spectroscopy

- Muonic hydrogen hyperfine structure 